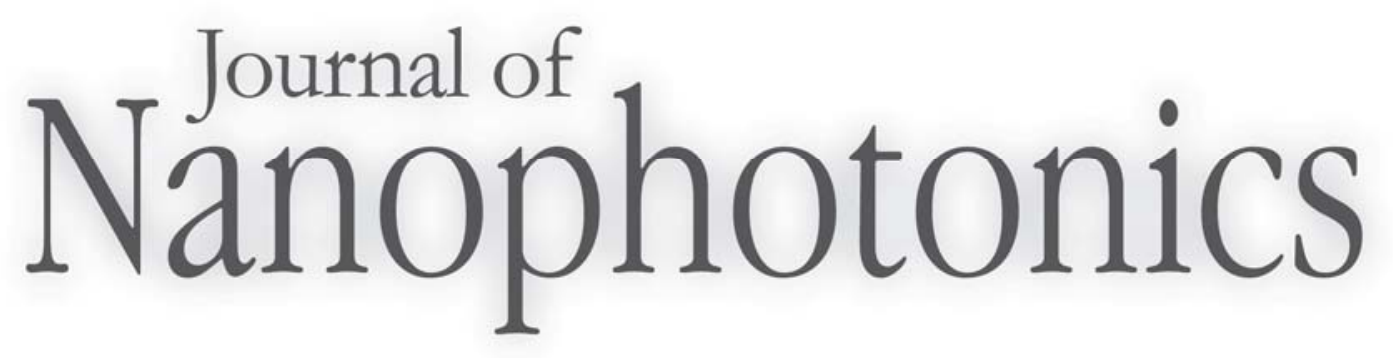

SPIEDigitalLibrary.org/jnp

\title{
Book Review: \\ Nanobiotechnology and Nanobiosciences
}

Reviewed by

Tihana Mirkovic and Gregory D. Scholes 


\section{BOOK REVIEW}

\section{Nanobiotechnology and Nanobiosciences}

Claudio Nicolini, 380 pages, ISBN 978-981-4241-38-0, Pan Stanford, Singapore (2009), $\$ 119.00$, hardcover.

Reviewed by Tihana Mirkovic and Gregory D. Scholes, University of Toronto, Department of Chemistry, Toronto, Ontario M5S 3H6, Canada, gscholes@chem.utoronto.ca

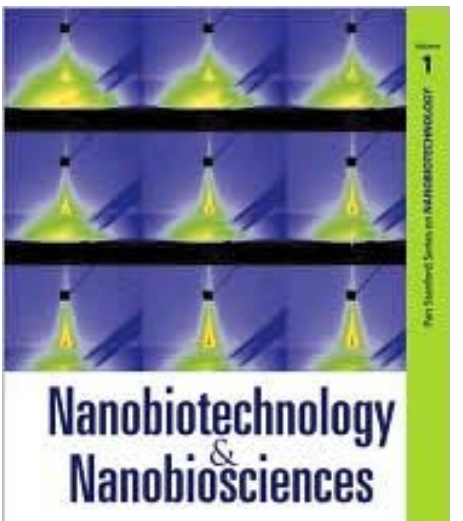

CLAUDIO NICOLINI

Nanoscience is such an incredibly broad subject that it is difficult to summarize in any single textbook. Nevertheless, Nicolini provides a concise and useful overview in Nanobiotechnology and Nanobiosciences. This book focuses on 'bio' applications of nanoscience, and touches on, for example, experimental approaches, health science, nanobioelectronics, photovoltaics, and nanobiocatalysis. The 'nanobio' trend is evident in these subject headings! Professor Nicolini, Director of the Nanoworld Institute in Genoa (http://www.nwi.unige.it/) and advisor to the Prime Minister of Italy, is an eminently qualified authority on nanobiosciences and this is conveyed in his writing.

The book describes many fascinating examples of applications, for example, how could natural photosynthetic reaction centers be used for photovoltaic applications? Other examples range from crystal formation to holography with halobacteria to the use of optical tweezers. The vast number and range of these examples is incredible, and they are carefully referenced in a comprehensive bibliography. In this fashion, Nicolini does an excellent job of presenting the myriad tools and applications developed in nanoscience, and he manages this without hype or overstatement.

The book is organized into four chapters, thereby chronologically introducing us to the full process of nanoscience research from the currently most widely applicable fabrication methods for nanomaterials and nanocomposites, to characterization and finally the development of nanoscale applications in health science and industry.

Chapter 1 gives an overview of nanostructures of biological origin and also explores nanomaterials derived through synthetic chemistry. Cells, proteins, genes and lipids are introduced in the context of nanotechnology through very specific case studies giving ample references to the reader to refer to, but the non-specialist might feel the lack of interconnectedness in the presentation between the various topics. Specific focus is placed on conductive polymers, carbon nanotubes, nanoparticles and their nanocomposites in the examples based on nanomaterials realized through chemical synthesis. The LangmuirBlodgett (LB) technique is highlighted throughout the text as one of the favorite methods for self-assembly of bionanomaterials, specifically illustrating the physical properties of LB films of proteins and lipid-protein complexes. A discussion on the implementation of nanochannelarray materials, such as the inorganic anodic nanoporous alumina matrix in protein array applications, wraps up the first chapter.

In Chapter 2, a large number of characterization methods relevant to nanoscale materials are described through a combination of introductory text and typical examples from the 
literature. These techniques include: surface potential, atomic force microscopy (AFM), nuclear magnetic resonance (NMR), ellipsometry, electrochemistry, infrared spectroscopy, nanogravimetry, biomolecular microarrays, biophysical information, mass spectrometry and synchrotron radiation. While the descriptions of each method are brief and can serve as an overview of characterization techniques, appropriate references point the reader to sources which provide more detail and can be useful to practitioners in the field.

In Chapter 3, Nicolini has taken on an almost impossible task to condense the immense and diverse, sometimes seemingly loosely related areas of nanoscale applications in science and health into one chapter. For example, in the section on nanomedicine, photosynthetization of titanium dental implants are discussed, as well as the adaptation of carbon nanotubes for in vivo applications, such as drug delivery. We get to sense the flavor of the emerging field of nanooptics in the vividly presented section on optical tweezers. Other areas of discussion in this chapter include nanobiocrystallography, nanogenomics, nanoproteomics and cell nanobioscience.

Chapter 4 is a sort of culmination to the book, where particular emphasis has been placed on the scientific achievements of the Nicolini laboratory in the incorporation of nanobio systems in industrially relevant nanoscale applications related to electronics, energy and catalysis. Properties and performances are detailed for some nanoscale systems with commercial potential ranging from protein-based and organic nanosensors, resistors, wires, displays, photovoltaic cells, batteries, to bioreactors, to name a few.

In our opinion, the book will appeal to a broad readership. It is a splendid, accessible introduction to nanotechnology with an emphasis on the interface with biology. There is an immense amount of information presented in this text and the extensive list of references forms a good foundation for someone who wants to do more research in depth. The layout of the book might be well suited as a text for an already established graduate level course, as little introduction is provided in certain sections and previous familiarity with the "nanoworld" is assumed. 